\title{
The Mu2e crystal calorimeter
}

\section{Eleonora Diociaiuti ${ }^{* \dagger}$}

LNF - INFN and Tor Vergata University E-mail: eleonora.diociaiuti@lnf.infn. it

The Mu2e Experiment at Fermilab will search for coherent, neutrinoless conversion of negative muons into electrons in the field of an aluminum nucleus. The dynamics of this process is well modelled by a two-body decay, resulting in a mono-energetic electron with an energy slightly below the muon rest mass $(\sim 104.967 \mathrm{MeV})$. If no events are observed in three years of running, Mu2e will set an upper limit on the ratio between the conversion rate and the capture rate $\mathrm{R}_{\mu e} \leq 6 \times 10^{-17}$ (@ 90\% C.L.). This will improve the current limit of a factor of $10^{4}$ over previous experiments The experiment complements and extends the current search for $\mu \rightarrow e \gamma$ decay at MEG as well as the direct searches for new physics at the LHC. Indeed, such a charged lepton flavor-violating reaction probes new physics at a scale inaccessible with direct searches at either present or planned high energy colliders.

We briefly present the physics motivation for Mu2e, the current status of the experiment and the design of the muon beamline and the detector, devoting particular attention to the $R \& D$ phase, simulation and test carried out to prove the validity of the electromagnetic calorimeter configuration.

The 39th International Conference on High Energy Physics (ICHEP2018)

4-11 July, 2018

Seoul, Korea

* Speaker.

${ }^{\dagger}$ A footnote may follow. 


\section{The Mu2e experiment}

The goal of the Mu2e experiment [1] is to search for the neutrinoless, coherent conversion of muons into electrons in the field of a nucleus and to improve by four orders of magnitude the previous sensitivity set at $90 \%$ C.L. by the SINDRUM II experiment [2]. This corresponds to a limit on the ratio between the conversion and nuclear muon capture rates $R_{\mu e}<8.4 \times 10^{-17}$ ). Mu2e hopes to achieve a Single Event Sensitivity (SES) of $\sim \mathbf{3} \times 10^{-17}$ with $\sim 0.5$ event background.

The muon coherent conversion into electrons in the field of a nucleus is one of the most promising process to probe Charge Lepton Flavour Violation (CLFV) since it has a very clear signature [3]: a single conversion electron (CE) with a monochromatic energy slightly below the muon rest mass: $E_{e_{\text {conv }}}=104.97 \mathrm{MeV}$.

In Figure 1, the layout of the Mu2e experiment is reported. For a more detailed description of the experiment see [4].

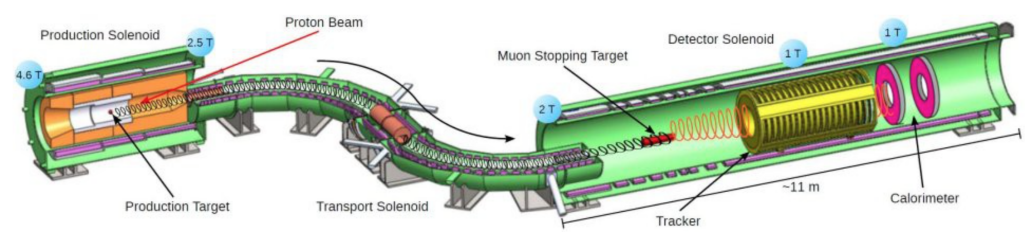

Figure 1: Schematic view of the experimental apparatus.

\section{Module-0 beam test results}

A large size prototype, called Module- 0 , was built using 51 crystals and each coupled to two custom Mu2e SiPMs (amplified by a prototype of the FEE boards). During the testing phase, a stable running temperature of $20{ }^{\circ} \mathrm{C}$ was kept on the SiPMs by using a cooling station and the designed cooling plate. The gain stability was monitored in the central crystal using a laser light. An exploded view of Module- 0 is shown in Figure 2.

A dedicated beam test was set up during May 2017 at the Beam Test Facility (BTF) in Frascati

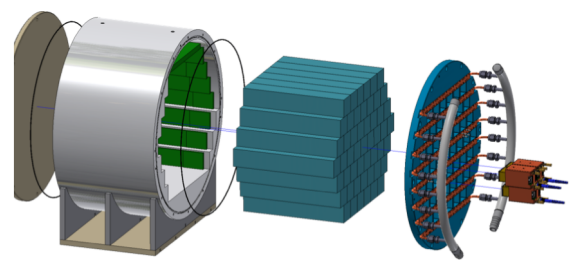

Figure 2: Module-0 components.

(Italy), exposing Module-0 to an electron beam with energy ranging from 60 to $120 \mathrm{MeV}$. Two configurations were studied during the test, with the beam impinging at: (i) $0^{\circ}$ with respect to Module-0 front face, to evaluate the calibration factors and study the resolution behaviour at different energies and (ii) at $50^{\circ}$ with respect to the calorimeter face, to simulate the expected average 
incidence angle of a CE in Mu2e. The charge was estimated by numerical integration of the waveform in a time window of $200 \mathrm{~ns}$. While a beam calibration was performed in the central region of the matrix, the whole Module-0 was calibrated also with cosmic rays. After the calibration, the energy deposited in all matrix was evaluated summing the energies collected in all the crystals, after correcting for baselines and for the proper calibration factors. In the left (right) part of Figure 3 the energy distribution for a $100 \mathrm{MeV}$ electron beam is shown in a orthogonal (tilted) configuration.
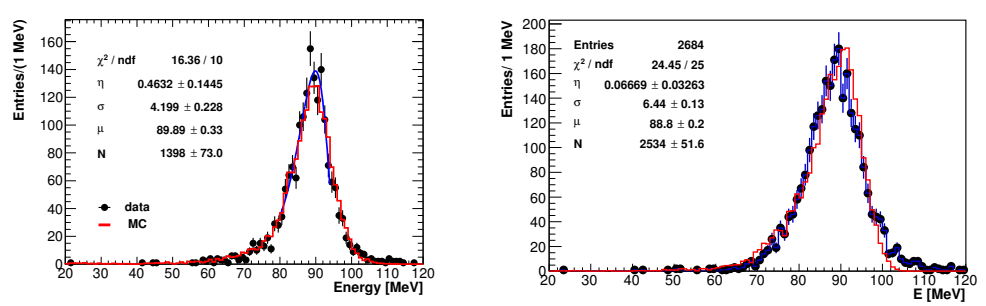

Figure 3: Left: Energy deposit in the whole Module-0 with a orthogonal run at different beam energies. Right: Energy deposit in the whole Module- 0 with a tilted run at different beam energies.

An energy resolution of about 5\% (7\%) was obtained in the orthogonal (tilted) configuration as shown in Figure 4.The fit fuction used is: $\frac{\sigma_{E}}{E}=\frac{a}{\sqrt{E}[\mathrm{GeV}]} \oplus \frac{b}{E[\mathrm{GeV}]} \oplus c$, where the coefficient $a$ represents the stochastic term, $b$ the noise term and $c$ the constant term. Since the fit was insensitive to the stochastic therm, $a$ was fixed to $0.6 \%$ estimating it by the light yield contribution of 40 $\mathrm{pe} / \mathrm{MeV}$. The energy resolution deteriorated in the tilted run because of the increasing of fluctuation in the leakage term. The signal time is determined by fitting the leading edge of the waveform with

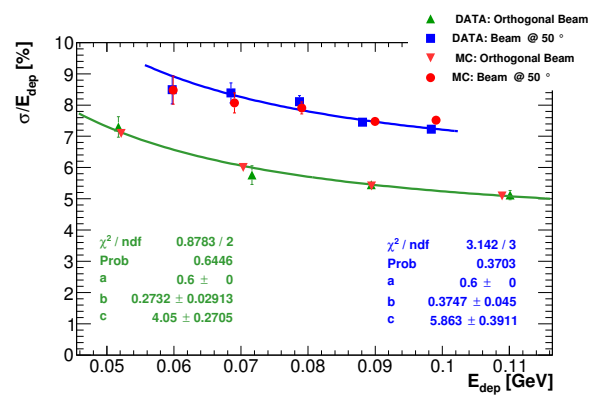

Figure 4: Energy resolution as a function of the energy deposit in the Module-0 in the orthogonal (blue) and tilted (green) configuration and comparison with the MC expectation.

an asymmetric log-normal function[5] and fitting the constant fraction at 5\%. For Module-0 the time resolution was measured using the time difference between the signals of two SiPMs that collect light from the same crystal. Figure 5 (Right) shows the resulting distributions for electrons impinging at $0^{\circ}$ the central crystal. The time resolution was deduced by applying a Gaussian fit on the distributions and dividing by $\sqrt{2}$ the $\sigma$ of the distribution to take into account the contribution of the two SiPMs. In Figure 5 (left) the time resolution as a function the deposited energy in the highest energetic crystal is reported. 

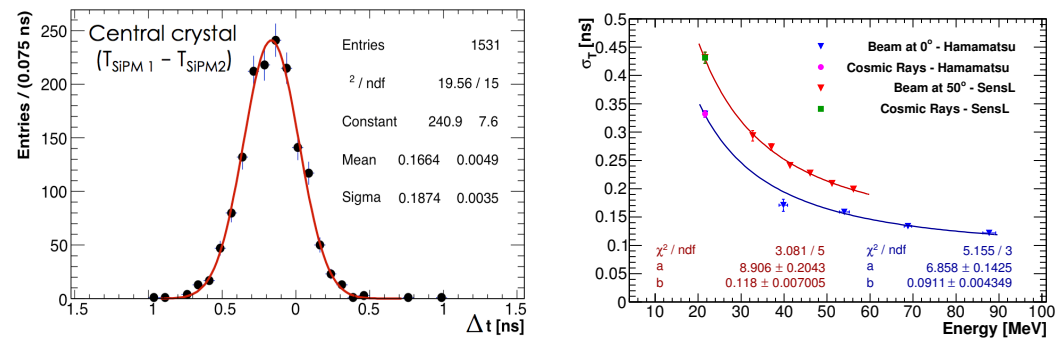

Figure 5: Right: Time diffeence between the two Hamamatsu SiPMs reading out the central crystal, when a $100 \mathrm{MeV}$ beam enters perpendicularly. Left: Time resolution as a function of the deposited energy in the highest energetic crystal

\section{Conclusion}

The Mu2e experiment will exploit the world highest intensity muon beams of the Fermilab Muon Campus to search for CLFV, improving current sensitivity by a factor $10^{4}$. The Mu2e calorimeter is a state of the art crystal calorimeter with excellent energy $(<10 \%)$ and timing $(<$ $500 \mathrm{ps}$ ) resolutions and a good pileup discrimination capability.

A large size prototype, called Module-0, was built in April 2017 to test integration and assembly procedures and confirm simulated performances obtaining a goot time and energy resolution in agreement with the mu2e expecification.

\section{Acknowledgment}

We are grateful for the vital contributions of the Fermilab staff and the technical staff of the participating institutions. This work was supported by the US Department onf Energy; the Italian Istituto Nazionale di Fisica Nucleare; the US National Science Foundation; the Ministry of Education and Science of the Russian Federation; the Thousand Talents Plan of China; the Helmholtz Association of Germany; and the EU Horizon 2020 Research and Innovation Progam under the Marie Sklodowska-Curie Grant Agreement N.690385. Fermilab is operated by Fermi Research Alliance, LLC under Contract No. De-AC02-07CH11359 with the US Department of Energy, Office of Science, Office of High Energy Physics.

\section{References}

[1] L. Bartoszek et al., Mu2e Technical Design Report, arXiv:1501.05241

[2] W. Bertl et al. A search for $\mu-$ e conversion in muonic gold. EPJ C, 47(2):337-346, 2006

[3] F.Grancagnolo et al., Nucl.Phys.Proc.Supp. 248-250, p 1-146 (2014).

[4] G. Pezzullo, Mu2e: A Search for Charged Lepton Flavor Violation in Muon-Electron Conversion with a Sensitivity $<10^{-16}$, same proceeding serie.

[5] C. Grupen, Particle detectors, Cambridge Monographs on Particle Physics, Nuclear Physics and Cosmology, 239-240, 1996 